JPE 10-5-3

\title{
Development, Implementation and Experimentation on a dSPACE DS1104 of a Direct Voltage Control Scheme
}

\author{
Ali Hmidet ${ }^{\dagger}$, Rachid Dhifaoui*, and Othman Hasnaoui* \\ $\dagger *$ RME Research Group, National Institute of Applied Sciences and Technology (INSAT), Tunis, Tunisia
}

\begin{abstract}
This paper proposes and develops a new direct voltage control (DVC) approach. This method is designed to be applied in various applications for AC drives fed with a three-phase voltage source inverter (VSI) working with a constant switching time interval as in the standard direct torque control (DTC) scheme. Based on a very strong $\min (\max )$ criterion dedicated to selecting the inverter voltage vector, the developed DVC scheme allows the generation of accurate voltage forms of waves. The DVC algorithm is implemented on a dSPACE DS1104 controller board and then compared with the space vector pulse width modulation technique (SVPWM) in an open loop AC drive circuit. To demonstrate the efficiency of the developed algorithm in real time and in closed loop AC drive applications, a scalar control scheme for induction motors is successfully implemented and experimentally studied. Practical results prove the excellent performance of the proposed control approach.
\end{abstract}

Key Words: Direct Voltage Control (DVC), dSPACE DS1104, Induction Machine (IM), Open loop and Closed loop experimental tests, Space Vector Pulse Width Modulation (SVPWM)

\section{INTRODUCTION}

During the last two decades, AC variable speed drives have demonstrated increasing success. This is the result of technological progress in power inverters and their very fast and accurate drivers on the one hand and progress in digital signal processors such as DSP and dSPACE kits on the other. Various control schemes are in fact built, experimentally studied and commercialised for industrial purposes. Field oriented control (FOC) and direct torque control (DTC) are typical examples [1]-[4].

All of the available FOC versions require a speed sensor and their machine voltage is ensured by a power inverter governed by the space vector pulse width modulation strategy (SVPWM) whose switching period is always in the range of 100 to 200 microseconds [1]. Due to the modulation principle, the actual switching times of the inverter IGBT's are variable in this range and can therefore reach very small values. The current waveform is then sufficiently smooth. Torque and speed rising times in the closed loop operating points are about the rotor time constant.

By principle, DTC schemes are speed sensorless [3]. Furthermore, DTC does not require switching time modulation. Inverter IGBT's are in fact commanded with a constant

Manuscript received Mar. 2, 2010; revised Jun. 21, 2010

† Corresponding Author: hmidet.ali@email.ati.tn

Tel: +216-23-227-117, Fax: +216-71-704-329, INSAT

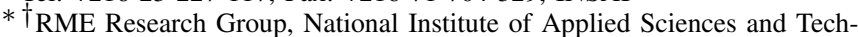
nology, Tunisia switching time that generally lies within the range of 25 to 50 microseconds. The torque and stator flux magnitude are realised by adequately selecting a voltage vector among those available in the inverter. The DTC structure is simple when compared to FOC. Furthermore, the torque response is very fast. The main drawback of DTC is the significant current and torque ripples if the selected voltage vector is not the best one.

Because the DTC option [5], [6] is the largest control scheme considered in our scope, the development of a voltage control strategy that works with a constant switching time is proposed in this paper. Consequently the aim of this paper is to obtain a suitable reference voltage vector directly with no need for space vector modulation. The developed method, here labelled DVC (Direct Voltage Control), is compared with the SVPWM technique, which is considered as a reference. The comparison is realised in an open loop control structure using an induction motor as a power process. Furthermore, practical implantation is realised on a dSPACE DS1104 controller board.

This paper is organized as follows. First, a brief description of the SVPWM technique is presented in section II. Then the theoretical background of the proposed DVC is developed and discussed in section III. An interesting point of the developed scheme is that the voltage vector selection is based on very strong $\min (\max )$ criterion. Moreover, we detail in the same section the fundamental steps of the algorithm and the Simulink structure to be implemented in the dSPACE DS1104 environment. The detailed structure of our test bench is given 


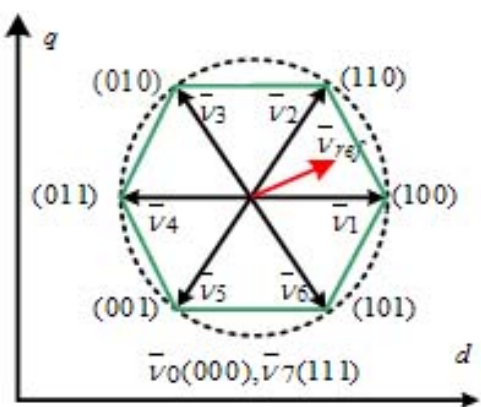

Fig. 1. Inverter voltage vectors in Concordia's reference frame.

in section IV. Section V presents and comments on the results of the comparison between the DVC and the SVPWM in an open loop running system. This study considers a lot of factors such as the quality of the waveforms, the harmonic spectrum, the losses, etc. Finally, we present in section VI the results of the DVC in the case of a closed loop scalar control structure. To ensure a good speed response, a PI controller anti-windup supplementary loop is used. Several experimental results are presented and discussed and some concluding remarks are outlined.

\section{Summary of Space Vector PWM Modulation TECHNIQUE}

The most accurate technique for obtaining an adequate voltage vector for variable speed applications of $\mathrm{AC}$ machines is surely the space vector approach [7], [8]. The space voltage vector approach is a two-components-based complex variable whose magnitude and angle are varied in time according to a desirable operating point; the stator flux magnitude and the machine speed for example.

For a lossless-admitted three phase voltage inverter, the output voltage is strictly defined by the DC bus voltage $\left(V_{d c}\right)$ supplying the inverter and the logical state of the three highest IGBT's $\left(c_{1}, c_{2}, c_{3}\right)$ in its configuration. There are only eight possible different logical combinations of $\left(c_{1}, c_{2}, c_{3}\right)$ leading to six active voltage vectors and two zero voltage vectors. It is well known that for a specified switching combination of $\left(c_{1}, c_{2}, c_{3}\right)$, the space vector of the inverter output voltage can be expressed as follows, where $k$ is an integer indicating the switching combinations [9], [10].

$$
\left\{\begin{array}{rlrl}
\text { for } k & =1,2, \ldots, 6: & & \bar{v}_{k}=V e^{j \theta v k} \\
V & =\sqrt{\frac{2}{3}} V_{d c} & & \theta_{v k}=(k-1) \frac{\pi}{3} \\
\text { for } k & =0 \text { and } 7: & & \bar{v}_{k}=0
\end{array} .\right.
$$

Fig. 1 summarizes this model by indicating in each vector the associated combination of the command signals $c_{1}, c_{2}$ and $c_{3}$.

The voltage inverter response is then discrete. Therefore, to achieve a continuous voltage signal $\bar{v}_{\text {ref }}$ defined by the controller of the AC drive, we have to synthesize from the discrete inverter voltage a mean value of the considered time interval. This is achieved by the well known SVPWM technique, which approximates the reference instantaneous voltage $\bar{v}_{r e f}$ by a combination of the switching states corresponding to

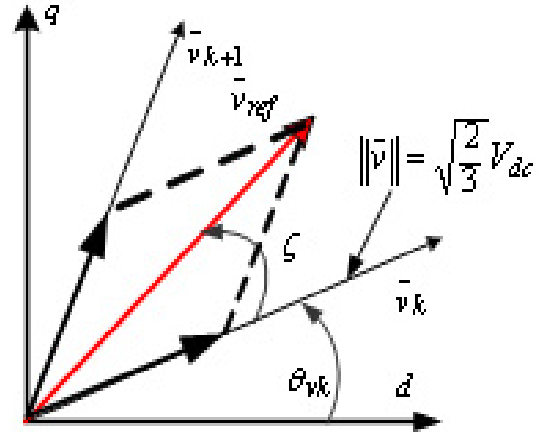

Fig. 2. Graphical construction of the reference voltage vector in Concordia's reference frame.

the basic space vectors. This means that it is required for the average of inverter voltage output to be equal to the reference voltage $\bar{v}_{r e f}$ for any period $T_{s}$. The solution is done by time modulating the two neighboring vectors in the sector where $\bar{v}_{r e f}$ is located, as indicated by Fig. 2.

For this purpose, it is assumed that the switching frequency is high and that the change in $\bar{v}_{r e f}$ over this period is small. The modulation scheme can be represented using the following equation [10]-[12]:

$$
\bar{V}_{\text {ref }}=\frac{1}{T_{s}}\left(T_{k} \bar{v}_{k}+T_{k+1} \bar{v}_{k+1}+T_{0} \bar{v}_{0}\right) .
$$

In this equation, $T_{k}, T_{k+1}$ and $T_{0}$ give the respective durations for which switching states corresponding to $\bar{v}_{k}, \bar{v}_{k+1}$ and $\bar{v}_{0}$ are applied. The voltage vectors $\bar{v}_{k}$ and $\bar{v}_{k+1}$ are the basic space vectors determining the sector containing $\bar{v}_{r e f}$. Vector $\bar{v}_{0}$ is the null switching vector activated for time $T_{0}$ to make up the rest of the switching period such that:

$$
T_{s}=T_{k}+T_{k+1}+T_{0} .
$$

According to Fig. 2 and equation 3, the solution satisfies equations 4 where $\rho$ and $\zeta$ are respectively the voltage ratio and the angle of $\bar{v}_{r e f}$ with respect to the considered sector.

$$
\begin{cases}T_{k}=T_{s} \rho \sin \left(\frac{\pi}{3}-\zeta\right) & T_{k+1}=T_{s} \rho \sin (\zeta) \\
\zeta \in\left[\begin{array}{ll}
0 & 60^{\circ}
\end{array}\right] & \rho=\frac{\sqrt{2} V_{r e f}}{V_{d c}}, \quad \rho \in\left[\begin{array}{ll}
0 & 1
\end{array}\right]\end{cases}
$$

The switching times $T_{k}$ and $T_{k+1}$ of the neighbouring voltage vectors $\bar{v}_{k}$ and $\bar{v}_{k+1}$ to the demanded vector $\bar{v}_{r e f}$ have a periodicity angle equal to 60 degrees and, antagonistic behavior. When $T_{k}$, for example, is near the maximum value, $T_{k+1}$ reaches a small value that can be less then a few microseconds. This means that the command will switch at a high local frequency leading to possible supplementary semiconductor losses. On the other hand, for some technological considerations, inverter drivers do not support a very low commutation time. In industrial processes, switching control uses the well known dead beat procedure to avoid switching times that are less than two microseconds for example.

\section{Fundamentals of the Proposed Direct Voltage CONTROL SCHEME}

Let us now consider that time is computed by a discrete clock having a sampling period $T_{d}$ that is chosen to be $N$ 


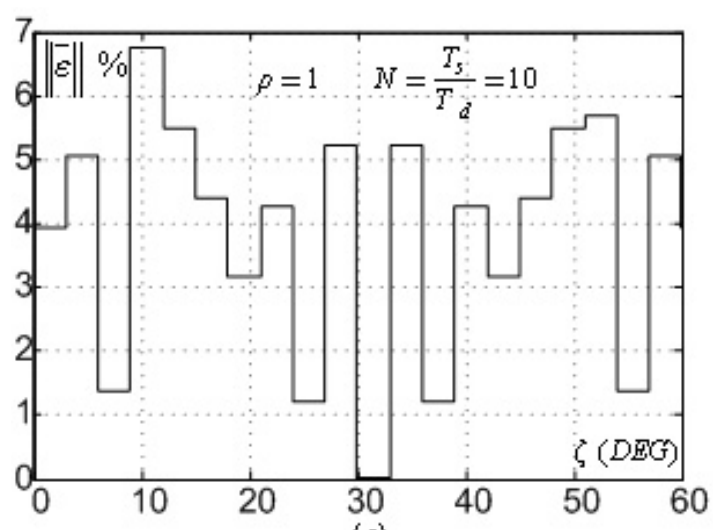

(a)

Fig. 3. Evolution of the voltage error versus reference voltage angle.

time smaller than the period $T_{s}$ used by the SPWM routine $\left(T_{s}=N T_{d}\right)$. During the SPWM period $T_{s}$, vector $\bar{v}_{1}$ will be used $N_{1}$ times while vector $\bar{v}_{2}$ will be used $N_{2}$ times. Integers $N_{1}$ and $N_{2}$ are defined by their rounded values that verify:

$$
\left\{\begin{array}{l}
N_{1}=\operatorname{round}\left(N \rho \sin \left(60^{\circ}-\zeta\right)\right) \\
N_{2}=\min \left(\operatorname{round}(N \rho \sin (\zeta)), N-N_{1}\right)
\end{array} .\right.
$$

Because the sum $\left(N_{1}+N_{2}\right)$ is not necessarily equal to $N$, at the end of the SPWM period $T_{s}$, there will be an error between the theoretical average voltage value and the actual synthesized one. The magnitude of this error is expressed by the following relation, showing its dependence on the integer $N$, the voltage ratio $\rho$ and the angle $\zeta$ :

$\varepsilon=\|\bar{\varepsilon}\|=\left\|\frac{\rho}{\sqrt{2}} e^{j \zeta}-\frac{N_{1}+\frac{N_{2}}{2}(1+j \sqrt{3})}{N}\right\|=f(\rho, N, \zeta)$.

Fig. 3 shows the evolution of the error magnitude $\varepsilon$ in $\%$ versus the voltage angle $\zeta$ when $\rho=1$ and $N=10$. As can be seen the error remains less than $7 \%$ on this angular interval. Because of the well known periodicity property of the switching time intervals of the SPWM, this error will remain less than $7 \%$ over the entire $360^{\circ}$ of space.

The variations in the defined error with respect to different values of $N$ and $\rho$ have also been studied and in each case the absolute maximum error has been retained. Fig. 4 summarizes the obtained results.

It is evident that if $N$ is great enough; the results theoretically converge with those of the SPWM solution. In other words, the errors will vanish. When analyzing the possibility of admitting some error level, it is obvious that admissible limits should be specified for $N$ and $\rho$. This means that by a compromise between the demanded magnitude of the voltage vector and the period $T_{d}$, an average vector voltage can be obtained with sufficient precision. In other words, instead of operating with the SPWM technique with a large period $T_{s}$, one of the available inverter voltage vectors can be directly selected after some shorter time interval $T_{d}$.

Let us designate with $n(t)$ the selected one among the seven inverter voltage vectors at the instant $t$. To force the average voltage to converge with the command value, we select a $n(t)$

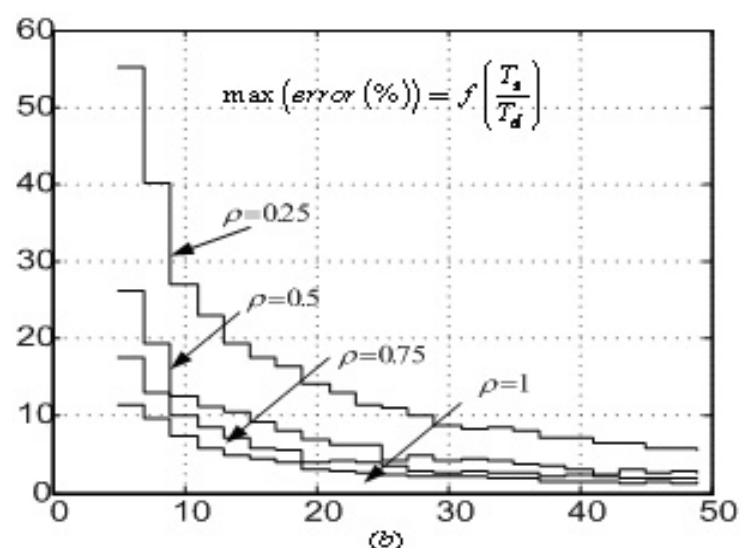

Fig. 4. Effect of voltage ration on the voltage error.

so that it minimises the voltage error along the time. This constraint is expressed by the following criterion:

$$
n(t) \rightarrow \min \int \bar{\varepsilon}(t) d t=\min \int\left(\bar{v}_{r e f}(t)-\bar{v}(n(t))\right) d t
$$

As the voltage vector is constant during the considered time interval, one can express the evolution of the voltage error by equation (8) and select the best inverter voltage vector according to the norm minimization as defined by equation (9).

$$
\begin{aligned}
& \bar{\varepsilon}\left(n\left(t_{k}\right)\right)=\varepsilon_{d}+j \varepsilon_{q}=\bar{\varepsilon}\left(t_{k}\right)+T_{d}\left(\bar{v}_{r e f}\left(t_{k}\right)-\bar{v}\left(n\left(t_{k}\right)\right)\right) \\
& n(t) \rightarrow \min \|\bar{\varepsilon}(n(t))\|=\min \left(\sqrt{\varepsilon_{d}^{2}(n(t))+\varepsilon_{q}^{2}(n(t))}\right) .
\end{aligned}
$$

The above minimization integral criterion must naturally respect at any time the $\mathrm{d}$ and $\mathrm{q}$ components. In some previous studies [5], [6] where this criterion has been used in the direct torque control of induction motors, it has been observed that in certain cases, this criterion can have the same value for two vectors voltages. This situation occurs when the vector error is located exactly between two consecutive voltage vectors, which results in a hesitation over the choice of the appropriate vector. The application of the norm criterion leads to the choice of the first vector encountered in the direction of trigonometry. Such a choice has no effect on the direct component of the error. Contrary to this, the inverse component of the error is affected. In fact, the sign of this component changes from one choice to another. To palliate at this, the norm criterion was transformed to a new criterion named $\min (\max )$. The objective of this criterion is to minimise the maximum between direct and the quadrature components of the voltage error. That is, the best voltage vector is selected according to:

$$
n \rightarrow \min _{n=1, \ldots, 7}(\varepsilon(n))=\min _{n=1, \ldots, 7}\left(\max \left(\left|\varepsilon_{d}(n)\right|,\left|\varepsilon_{q}(n)\right|\right) .\right.
$$

The direct voltage vector scheme is summarised by the following steps:

a. Choose a vector voltage and compute the new associated $\mathrm{d}$ and q components of the flux error with equation (9). The maximum absolute value of these components is retained and saved.

b. Repeat step a) for the other voltage vectors. 
TABLE I

MOTOR SPECIFICATIONS

\begin{tabular}{|c|c|c|c|}
\hline$R_{s}$ & $4 \Omega$ & $p$ & 1 \\
\hline$R_{r}$ & $4.5328 \Omega$ & $J$ & $0.0015 \mathrm{Kgm}^{2}$ \\
\hline$L_{s}$ & $0.4334 \mathrm{H}$ & $\omega_{r n}$ & $2830 \mathrm{rpm}$ \\
\hline$L_{r}$ & $0.4334 \mathrm{H}$ & $P_{n}$ & $1.5 \mathrm{~kW}$ \\
\hline$M$ & $0.399 \mathrm{H}$ & $V_{n}$ & $220 / 380 \mathrm{~V}$ \\
\hline
\end{tabular}

c. Sort the seven saved values in increasing order, select the first one and detect the associate index voltage $k$ as given by (10).

Fig. 5 gives a general view of this control scheme realized in the Matlab/Simulink environment.

\section{A Brief Description Of The Experimental PROCESS}

The previously developed algorithm is implemented and tested in an experimental environment. Fig. 6 summarizes the general architecture of our benchmark. A photograph of the experimental setup is shown in Fig. 7. The benchmark consists of:

- A dSPACE DS1104 controller board with a TMS320F240 slave processor and an ADC interface board CP1104. The DS1104 board is installed in an Intel(R) Pentium(R) D CPU $3.4 \mathrm{GHz}$ PC for software development and results visualization.

- A two-pole induction motor (the parameters are listed in table 1) loaded with a magnetic particle brake capable of producing a torque proportional to its current excitation. For speed measurement purposes, the machine shaft is coupled to an incremental encoder having 360 pulses per revolution.

- A three-phase VSI whose dc bus voltage is generated by a rectifier connected to a $400 \mathrm{~V}-50 \mathrm{~Hz}$ AC electrical source.

- A 4-channel, $150 \mathrm{MHz}$ digital oscilloscope for current and voltage registration and a 2-channel, $20 \mathrm{MHz}$ ana$\log$ oscilloscopes for online visualization of the desired variables.

- Some devices for current and voltage measurement. All of these devices are sensors that are LEM-based and calibrated so that the obtained analogue signals should remain in the range of 0 to $\pm 10 \mathrm{~V}$ as required by the $\mathrm{A} / \mathrm{D}$ converters of the dSPACE.

The benchmark includes a lot of other devices such as standard measurement equipment, analogue signal filters, analogues circuits defining the dq voltage and current components, etc.

\section{A COMParison OF DVC to SVPWM IN AN OPEN LOOP REGIME}

To evaluate the performance of the proposed DVC scheme developed in section III it is compared to the conventional SVPWM technique in a real time application. The SVPWM modulation technique remains the most adequate reference to show the appropriateness and effectiveness of this work. These two approaches have been implemented in the Simulink environment working in real time by a dSPACE DS1104. The two approaches are then successively tested while supplying an induction machine working in an open loop. Fig. 8 gives a general view of the studied structure. The same frequency $\left(\omega_{s}=100 \pi \mathrm{rad} / \mathrm{s}\right)$ and voltage ratio $(\rho=0.8)$ of the applied reference voltage vector, the same induction motor, the same load, etc. have been respected. The sampling times used in the real tests are $T_{s}=50 \mu s$ for the DVC approach and $T_{s}=200 \mu s$ for the SVPWM technique, respectively.

Fig. 9 through 12 summarize the generated practical results. Curves indicated by 'a)' correspond to the case of the SVPWM technique considered here as a reference method and curves indicated by 'b)' correspond to results of the proposed DVC scheme.

Fig. 9 furnishes the direct component of the instantaneous value and the moving average value (local mean value) of the stator voltage for both the SVPWM and DVC approaches. We observe without ambiguity that the average value generated by the DVC scheme is sinusoidal as well as the SVPWM method. This successful result is confirmed by the good current waveforms shown in figure 10(b). Note also in figure 10(b) the very accurate perpendicularity between the dq current components of the DVC scheme.

The current waveforms obtained by the two methods (Fig. 10) are also treated in terms of harmonic decomposition. The harmonic spectrums and the THD values are presented in Fig. 11. We can see in this figure that the current THD values obtained by the two methods are very similar. This implies that the proposed scheme has practically the same potentialities as the SVPWM technique. This is a very promising result. However, this optimistic conclusion should be considered very carefully. In fact, intensive study of this problem by including the effects of frequency and voltage ratio ranges should be accurately carried out. A future work will discuss this point with more detailed information. For the moment, we conclude that the developed approach largely satisfies the tracked objective. Fig. 12 supports this satisfactorily. It shows a front view of the used digital oscilloscope tracking the $\mathrm{d}$ and q components of the sinusoidal reference voltage and the actual filtered voltage. The filter used is an analogue adequate RC circuit.

System losses are an important factor not only from an energy economy point of view but also when considering possible excessive heating, which can damage the circuit. These losses are globally dominated by joule losses, iron losses and switching losses in the voltage inverter. The former type of losses is highly dependent of the number of commutations in a given time interval. In this sense, we have counted the number of commutations over a time interval equal to $100 \mathrm{~ms}$ for the two methods (standard SVPWM and the proposed DVC). This corresponds to 5 fundamental periods of $50 \mathrm{~Hz}$. It should be noted here that the count of the commutations is realized not on the command signals of the voltage inverter but on the actual voltage supplying the induction machine (the actual output of the inverter). For this purpose, the machine voltage is saved during $100 \mathrm{~ms}$ with a digital oscilloscope (MTX 3354) giving 50,000 samples. This means that we have one sample every two microseconds which is large enough 


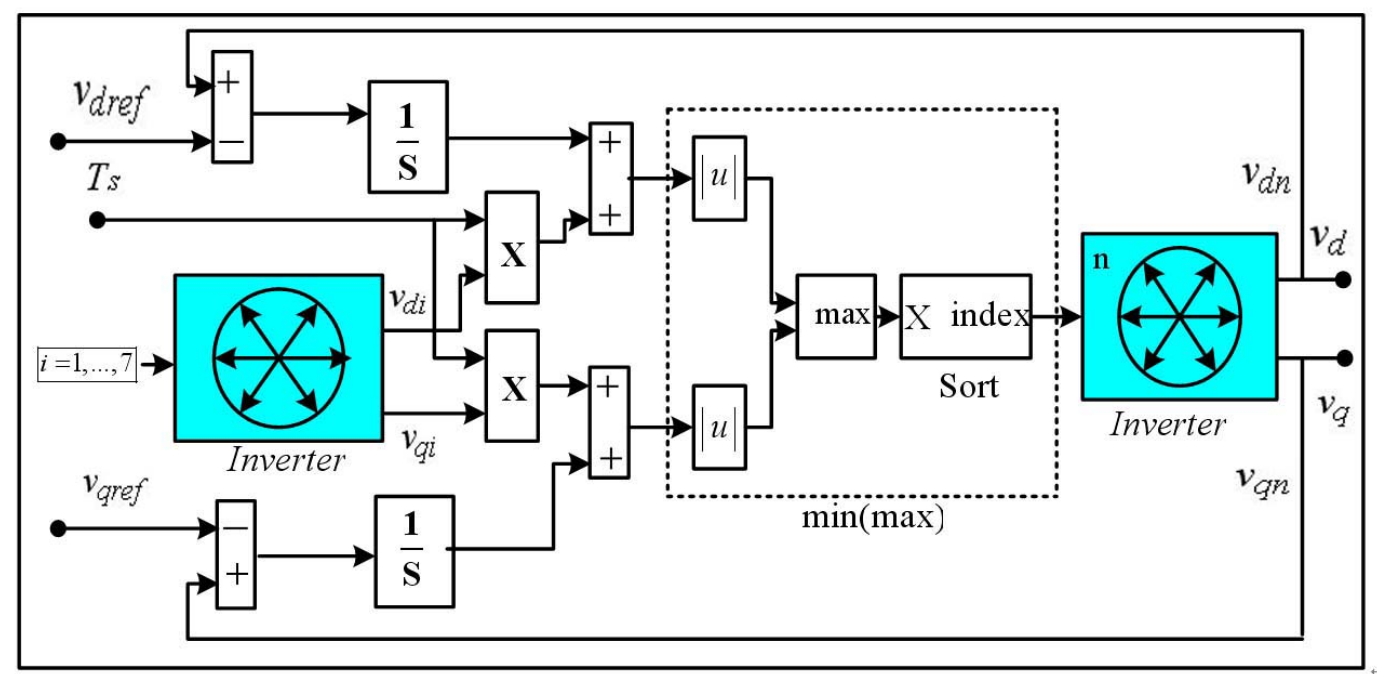

Fig. 5. Structure of the defined $\min (\max )$ DVC criterion.

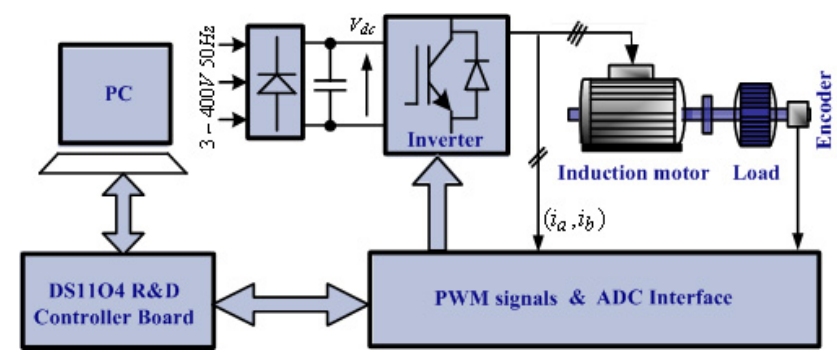

Fig. 6. General structure of the used experimental setup.

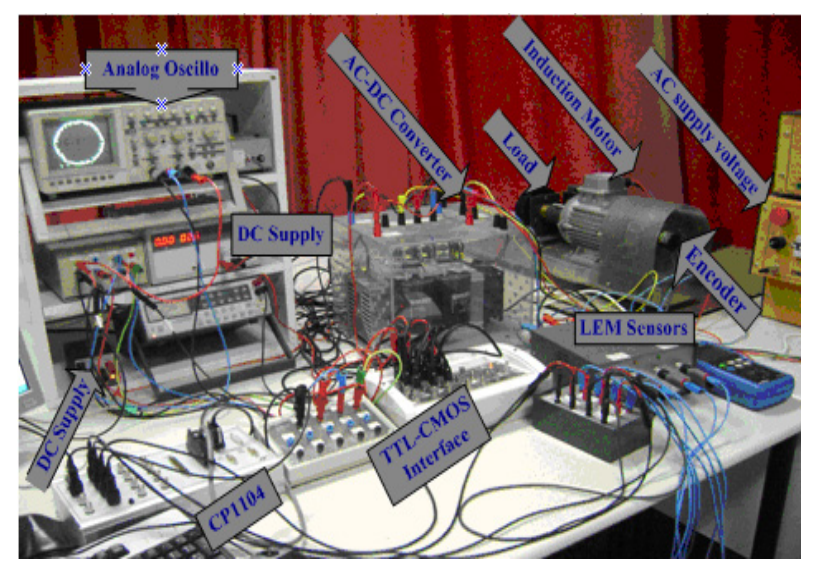

Fig. 7. Photograph of the experimental setup.

to have good accuracy in impulsions counting. The obtained result shows that the number of commutations is reduced from 1309 with SVPWM to 1083 with DVC. This is a reduction of $17.27 \%$. This implies that the switching losses in the inverter are consequently reduced by about $17.27 \%$.

This study shows that the developed approach has a lot of interesting and advantageous points such as:

$\checkmark$ The proposed DVC scheme does not require the use of a professional space vector pulse width modulation (SVPWM) module, which is in general a built in generator, because it requires a very fast computing time.

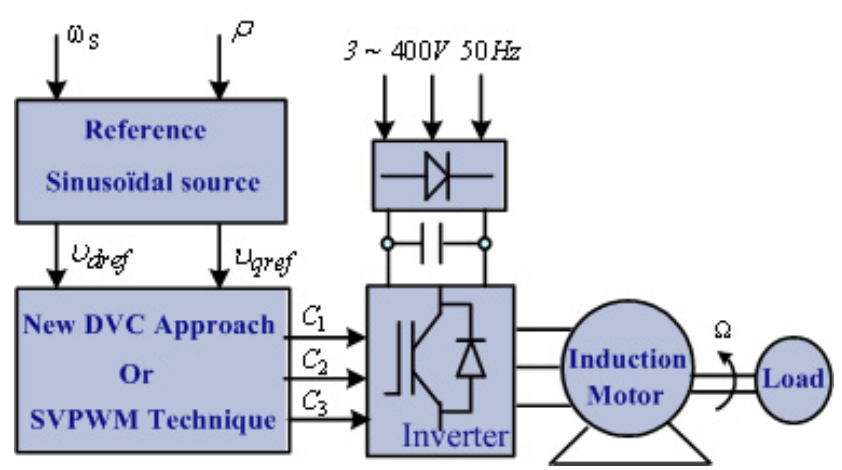

Fig. 8. Open loop DVC or SVPWM technique.

$\checkmark$ Digital implementation of DVC is relatively easy and therefore possible on non expensive circuits such as PICbased or DSP-based development kits. An immediate benefit can be found in developing the sector of industrial informatics in laboratories.

$\checkmark$ Working with constant switching time avoids the use of high quality power electronics components. This leads to the possibility of building non sophisticated and low cost inverters based on the example for classical transistors.

$\checkmark$ Minimizing the number of commutation keys, consequently minimizing the switching losses in the inverter.

\section{Checking The DVC Approach In Closed Loop REGIME HECKING}

\section{A. Presentation of the implemented control structure}

The study presented and commented on in the previous section for the proposed DVC scheme was restricted to steady state operating points. To test and check the performance of this scheme in closed loop control, the scalar constant voltage-frequency ratio (CVFR) technique was implemented. A well known version of the CVFR technique uses the measured machine speed signal and a PI controller to define the electrical frequency reference, and the voltage reference is considered proportional to this frequency [13], [14]. To 

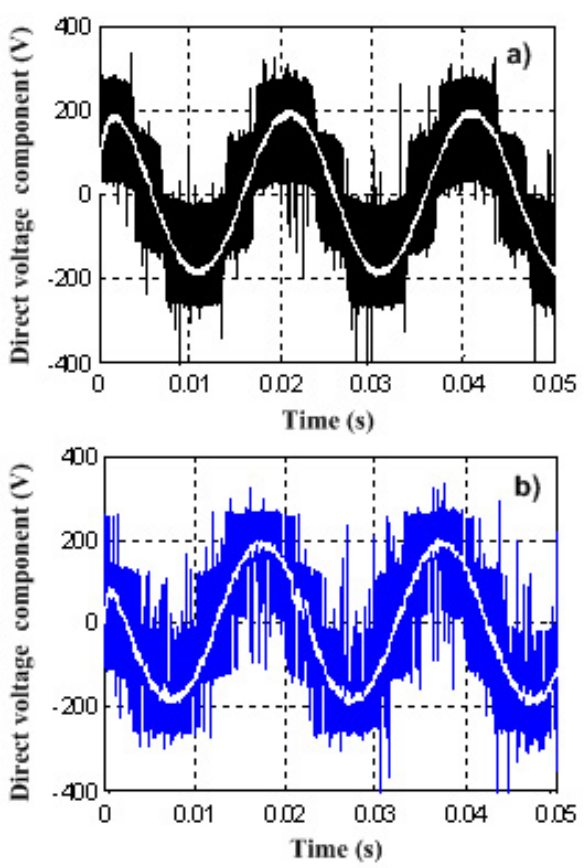

Fig. 9. Direct Voltage component.
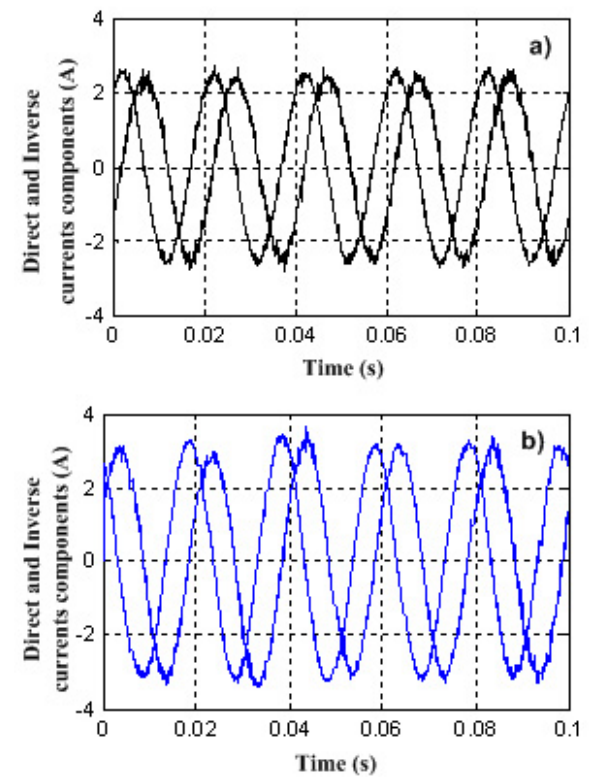

Fig. 10. Direct and quadrature currents components.

keep the voltage reference in the permitted practical limit, a saturation block is added as indicated by figure 13, which sketches the implemented control structure. In this structure there is a reference block (voltage and frequency commands) defined by the CVFR and the DVC block that generates the power inverter command signals as previously introduced.

In practical implementation, the main drawback of the CVFR procedure lies in the effect of the stator-voltage drop especially in low speed operation. The voltage drop at low frequencies has the same order of magnitude as the computed voltage and it makes the method inadequate for the low speed region. This problem can be partially compensated for by
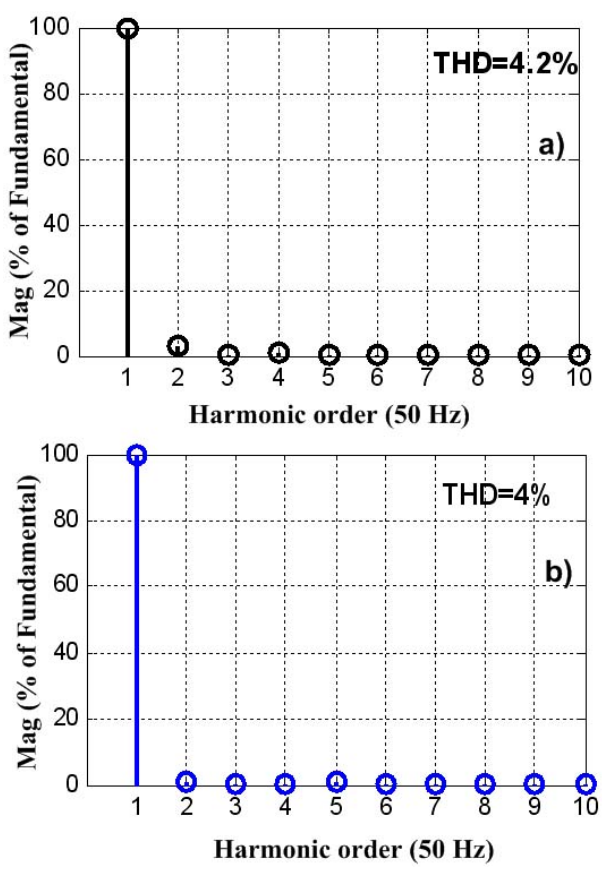

Fig. 11. Stator current harmonic spectrum.

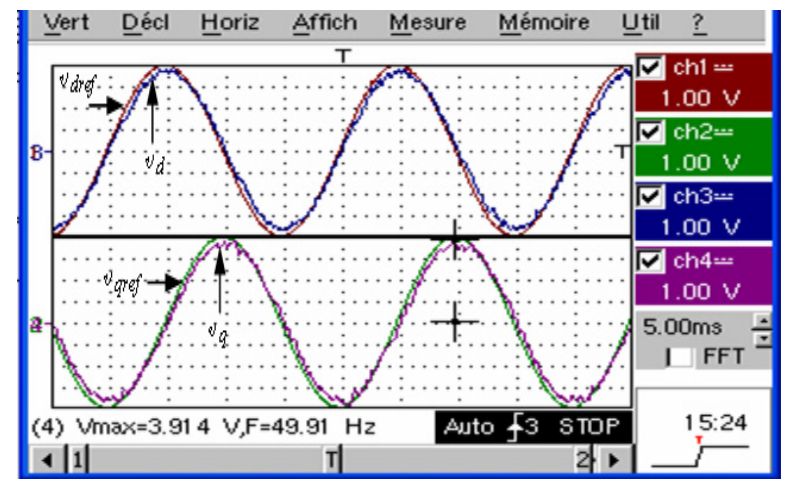

Fig. 12. Comparison of reference voltage and actual filtered voltage obtained by DVC scheme.

according an approximate relation, instead of the nonlinear theoretical relation. This is why the following linear voltagefrequency dependency was used. In this equation, $V_{0}$ is the boost voltage and $K_{v}$ defines the slope that corresponds to the nominal stator flux value.

$$
V_{s}=V_{0}+K_{v} \omega_{s} .
$$

It is also important to note that we have enhanced this control structure by introducing an improvement upon the classical PI controller. In severe transient regimes, as induction machines start up for example, the saturation of the manipulated variable can introduce a phenomenon that causes a racing of the integral action, which is likely to deteriorate the performance of the system or even to destabilize it completely. To overcome this phenomenon, an internal anti-windup loop was added and implemented to the digital PI controller [15]. A Simulink model implementing the PI controller with the anti-windup scheme is shown in Fig. 14.

The source of the feedback to the integrator is the difference between the input (upstream value) and the output 


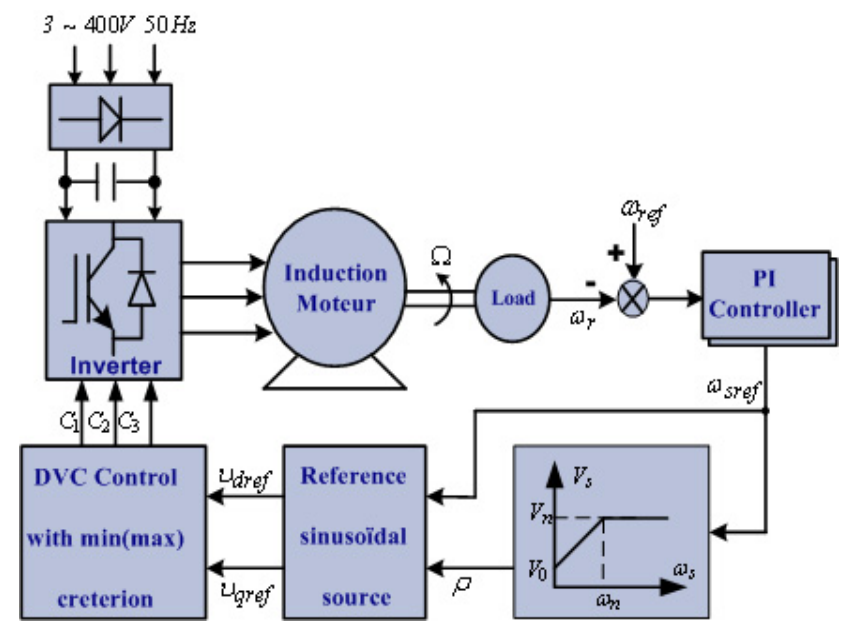

Fig. 13. Block diagram of closed loop scalar control with DVC approach.

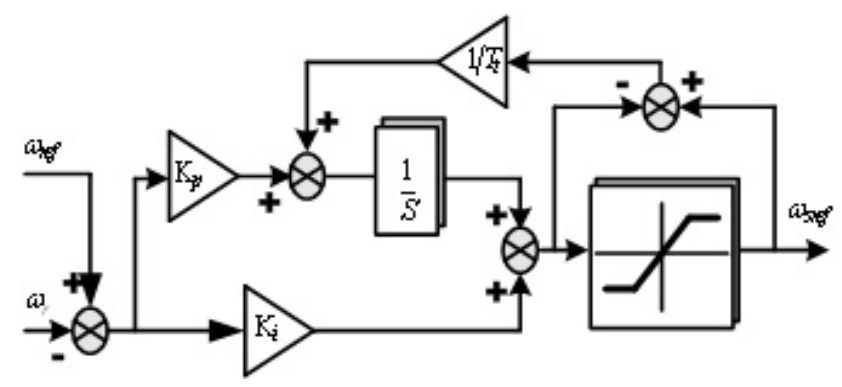

Fig. 14. Reference frequency generator with anti-windup PI controller.

(downstream value) of the saturation block. If "windup occurs" and the control signal becomes larger than the saturation limit, the difference becomes negative. This negative value is passed through a gain block (tracking time constant $T_{t}$ ) before arriving as feedback to the integrator.

\section{B. Results and comments}

To evaluate the performance of the implemented control structure, a series of measurements has been taken. These measurements correspond to a perturbation scenario which is made up of three cases. Graphical results shown by figures 15 to 17 are obtained using the Control Desk ${ }^{\mathrm{R}}$ graphical user interface provided in a dSPACE DS1104

Case i): The induction machine is initially running at a constant speed reference $(157 \mathrm{rad} / \mathrm{s})$ and under a no load regime. From this situation, we apply a load torque of $3 \mathrm{Nm}$ during a time interval of $10 s$ and then we remove the load. This case corresponds to a step up and a step down torque perturbation. The motor load is a magnetic powder brake that generates a torque proportional to the dc excitation current.

Fig. 15 corresponds to this case and presents time responses respectively of:

- the stator reference pulsation $\omega_{\text {sref }}$ at the output of the anti wind-up PI controller (curve a: red color),

- the electrical rotor speed $\omega_{r}$ captured by a speed sensor using an incremental encoder having 360 pulses per revolution (curve b: green color),
- the voltage duty ratio defining the reference value for the stator voltage magnitude (curve c: blue color).

It can be easily seen that this torque perturbation has a very small effect on machine speed. In fact, this variable has practically the same value as in the steady state. Only a very small and dumped variation occurs at the torque rising and decreasing. This implies that the anti-wind up PI controller works successfully and that the machine speed converges to its reference value. From figure 15 we have evaluated the machine speed deviation in percent with respect to the reference value. This deviation is negligible since it remains less then $5 \%$.

The most important effect due to the torque perturbation appears on the stator pulsation and the voltage magnitude. On the basis of the classical theory of induction machines and in particular for the practical operating area of the speed-torque curve, it is well known that the torque is proportional to the frequency and proportional to the square of the voltage. These properties explain why the stator voltage and the pulsation trajectories have the same shape as the torque command. Finally, as can be seen from figure 15 the system dynamics correspond to a settling time of about one second and a maximum overshoot of less then $5 \%$.

Case ii): The induction machine is initially running under a load torque producing a rotor speed of $94.2 \mathrm{rad} / \mathrm{s}$. The torque of the load is kept constant. A positive and then a negative step change of the speed reference is realized. The speed command is suddenly increased from $94.2 \mathrm{rad} / \mathrm{s}$ to $188.5 \mathrm{rad} / \mathrm{s}$ then decreases to the initial value of $94.2 \mathrm{rad} / \mathrm{s}$. Fig. 16 corresponds to this case and shows:

- The machine speed reference $\omega_{\text {ref }}$ (curve a: red color).

- The electrical rotor speed $\omega_{r}$ (curve b: green color).

- The stator reference pulsation $\omega_{s}$ (curve c: blue color).

- The voltage duty ratio (curve d: light blue color).

These results prove the good performance of the implemented control structure. In fact, the machine speed reaches its command without an undesirable lag or overshoot and therefore a very stable steady state point is obtained. Note also that the voltage duty ratio trajectory is highly similar to that of the stator frequency. The control structure is in fact built on the constant voltage-frequency ratio principle. On the other hand, an analysis of these signals has permitted an outline of some important conclusions. It is observed in particular that the dynamic behavior is characterized by a rise time of about $0.4 s$ a peak time of $0.6 s$, and a settling time that practically corresponds to $2 s$. The maximum overshoot is estimated at $4.5 \%$ for a no load regime.

Case iii): This case corresponds to a change in the speed reference executed exactly as it was for the previous case. The only difference is that the time saving is reduced and the number of samples is increased to observe, in more detail, the stator current signal. Fig. 17 corresponds to this case and furnishes:

- The voltage duty ratio (curve a: red color).

- The instantaneous stator current signal (curve b: blue color). 


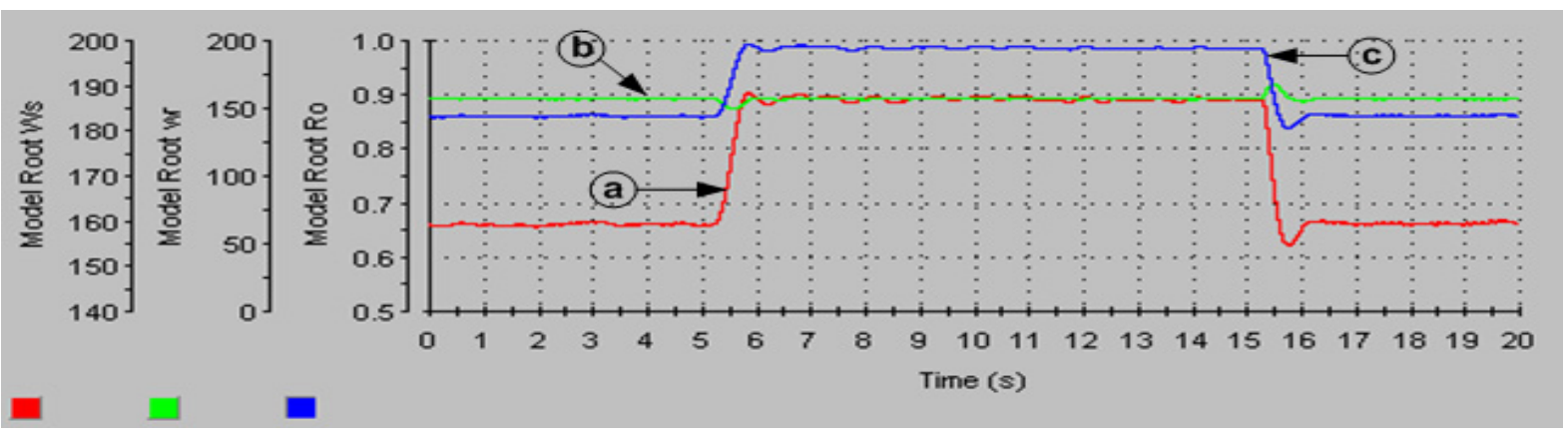

Fig. 15. System response for case i)

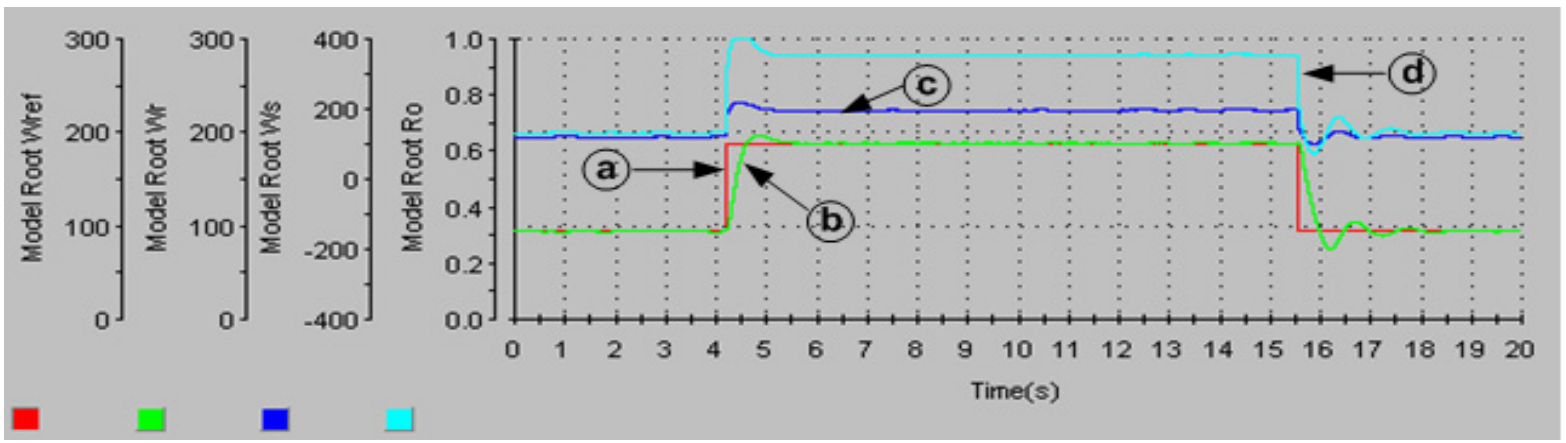

Fig. 16. System response for case ii)

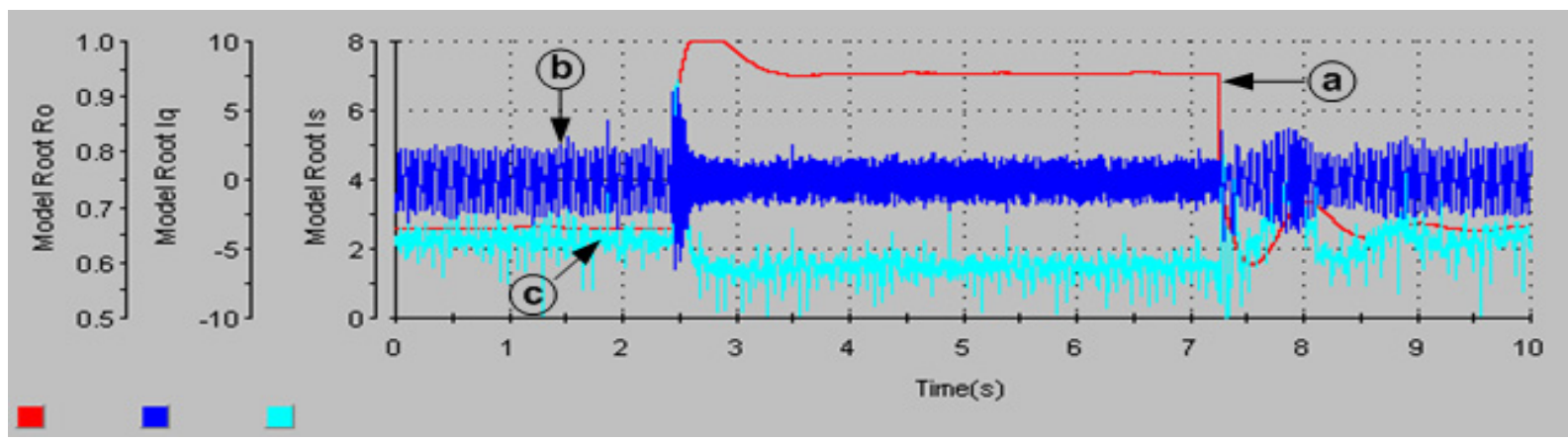

Fig. 17. System response for case iii)

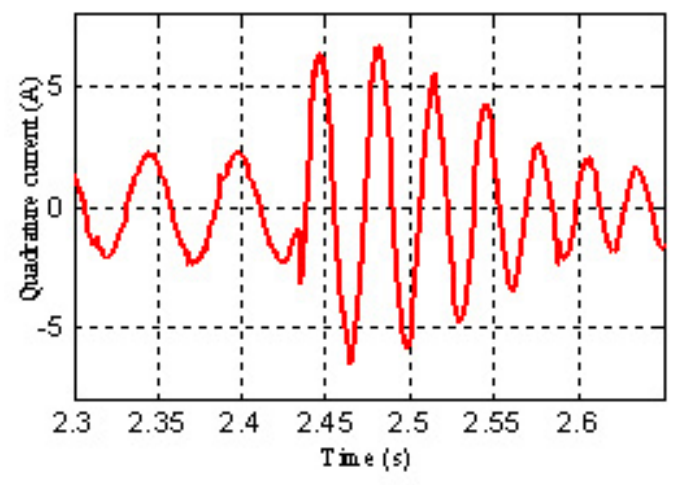

Fig. 18. A zoom in instantaneous stator current at rapid speed change.

- The stator current magnitude (curve c: light blue color). It is observed that the stator current magnitude decreases due to an increase in the stator frequency. On the other hand, it is also observed that stator current dynamics are very fast. Fig. 18 gives a zoom in of the current trajectory at the instant of a very sensitive machine speed change. The stator current trajectory changes rapidly from a sinus curve to a new one.

\section{CONCLUSIONS}

A novel algorithm for voltage control is proposed in this paper. This algorithm, named direct voltage control (DVC), can be applied to AC drives fed with power inverters. Practical implementation on a digital signal processor such as a dSPACE is simple and easy. We believe that the major contribution of this approach is in making the principle of direct control simpler and more reliable. The direct voltage control scheme is built on a powerful minimisation criterion to select the best inverter voltage vector. By testing the generation of several voltage forms of waves, this criterion has led to very promising results. In open-loop control and in close-loop control the 
proposed DVC algorithm has been implement on a dSPACE DS1104 with a sampling time of $50 \mu \mathrm{s}$. Experimental results show the correctness and feasibility of the proposed algorithm. Moreover, it has been discovered through an analysis of the command signals that this algorithm can reduce the number of switching commutations and consequently the semi conductor losses.

\section{REFERENCES}

[1] Domenico C., Francesco P., Giovanni S. and Angelo T., "FOC and DTC: Two variable schemes for induction motors torque control," IEEE Trans. On Power Elect., Vol. 17, No. 5, Sep. 2002.

[2] Nalin K.M., Rannganath M. and M. Senthil K., "A survery on controlled AC electrical drives," Int. Journal of Elect. and Power Eng. Vol. 3, No. 3, pp. 175-183, May. 2009.

[3] Giuseppe S. Buja, and Marian P. Kazmierkowski, "Direct torque control of PWM inverter-fed AC motors-a survey," IEEE Trans. On Indust. Elect., Vol. 51, No. 4, Aug. 2004.

[4] Naceri F., Belkacem S. , Kercha M. and Benmokrane T.: "Performance analysis of field-oriented control and direct torque control for sensorless induction motor drives," Proceedings of the $15^{\text {th }}$ Mediterranean Conference on Control \& Automation, July 27-29, 2007.

[5] O. Hasnaoui, H. Brahmi, N. Hidouri andR.Dhifaoui: "Direct voltage and current control schemes applied to permanent magnet synchronous motor," ELECTROMOTION 2005, Sep. 2005.

[6] Hmidet A., Bacha F. and Dhifaoui R.: "Vectored direct torque control for induction motor under losses minimization," ICEM 2006, Sep. 2006

[7] Xing S. and Zhao K.: "Research on a novel SVPWM algorithm," ICIEA 2007: $2^{\text {nd }}$ IEEE Conference on Industrial Electronics and Applications, May 2007.

[8] Xiaowei Z.; Yongdong Li; Wensen W.: "A novel implementation of SVPWM algorithm and its application to three-phase power converter," IPEMC 2000, Vol. 3, No. 1, pp. 1104 - 1107, 2000

[9] Keliang Zhou and Danwei Wang : "Relationship between space-vector modulation and three-phase carrier-based PWM: A comprehensive analysis," IEEE Trans. Ind. Electron., Vol. 49, No. 1, Feb. 2002.

[10] Zhiyong Du and Xianfang Wang: "SVPWM speed governing system of induction motor based on DSP," IEEE EUROCON 2007, Sep. 2007.

[11] Berrezzek F. and Omeiri A. "A study of new techniques of controlled PWM inverters," European Journal of Scientific Research, Vol.32 No.1, pp.78-88, 2009.
[12] Z. Wang, J. Jin, Y. Guo, and J. Zhu: "SVPWM techniques and applications in HTS PMSM machines control," Journal of electronic science and technology of China, Vol. 6, No. 2, Jun. 2007.

[13] Rakesh parekh: "VF control of 3-Phase induction Motor Using Space Vector Modulation," Microchip technology Inc., AN955, 2005.

[14] Tianchai S. and Satean T., "T-DOF PI controller design for a speed control of induction motor," Proceeding of World Academy of Science, Engineering an Technology, Vol. 26-12-2007, 2007.

[15] Naceri F., Belkacem S. , Kercha M. and Benmokrane T.: "Performance analysis of field-oriented control and direct torque control for sensorless induction motor drives," Proceedings of the $15^{\text {th }}$ Mediterranean Conference on Control \& Automation, Jul. 2007.

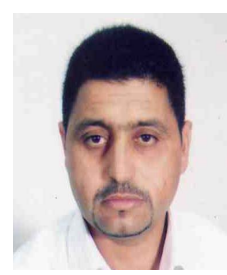

Ali Hmidet was born in Skhira, Tunisia in 1968. He received his M.S. in Industrial Informatics and Automatics from the National Institute of Applied Sciences and Technology (INSAT), Tunisia in 2004. He is currently pursuing his Ph.D. in Industrial Informatics at INSAT, Tunisia. He is now an Assistant Professor in the High Institute of Medical Technology at the University of Manar, Tunis, Tunisia. His research interests are electrical machines, power electronics, digital signal processing, renewable energy and AC drives.

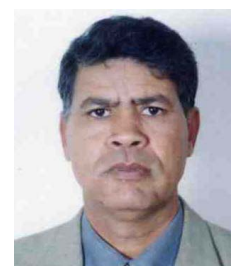

Rachid Dhifaoui was born in Kairouan, Tunisia in 1954. He received his "Doctorat d'Etat" in Electrical Engineering from ENSET, University of Tunis II, 1991. $\mathrm{He}$ is now a Permanent Professor at the National Institute of Applied Sciences and Technology (INSAT). He manages the studies of a Master Cycle in Industrial Informatics and Automatic Control. He is a manager of a research group (RME) working in the field of electrical machines and power systems.

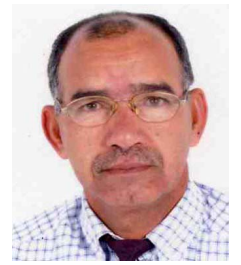

Othman Hasnaoui was born in Kef, Tunisia in 1954. He obtained his HDR in 2007 in Electrical Engineering from the Technical University ESSTT Tunisia. He is a Full Professor at ESSTT. His fields of interest include electrical machines, power electronics, control techniques and wind energy. 